\title{
ESTRATEGIAS DIDÁCTICAS PARA MEJORAR EL TRABAJO COLABORATIVO
}

\section{DIDACTIC STRATEGIES IN ORDER TO IMPROVE THE COLLABORATIVE WORK}

\author{
Eulalia Beatriz Becerra García ${ }^{1}$ \\ Gloria del Rocío Endara Prieto ${ }^{2}$ \\ Rita Piedad Edilma Poma Rojas ${ }^{3}$
}

\begin{abstract}
Recibido: 2019-08-10 / Revisado: 2019-09-10 / Aceptado: 2019-10-25 / Publicado: 2020-01-01
Forma sugerida de citar: Becerra-García, E. B., Endara-Prieto, G. y Poma-Rojas, R. P. (2020). Estrategias didácticas para mejorar el trabajo colaborativo. Retos de la Ciencia. 4(8), pp. 7685. https://doi.org/10.53877/rc.4.8.20200101.06
\end{abstract}

La presente investigación aborda el trabajo colaborativo en los estudiantes de tercer año de Educación General Básica en la Unidad Educativa "Quisapincha", ya que se evidenciaba desinterés por parte de los estudiantes a la hora de trabajar en equipos. En la investigación se utilizó una metodología cualitativa que permitió interpretar y caracterizar la realidad de la institución en cuanto al uso de trabajo colaborativo como metodología de aprendizaje, como instrumentos se utilizó la entrevista y encuesta, cuyos resultados demostraron la necesidad de diseñar y aplicar estrategias didácticas colaborativas como juegos, cantos, trabajos manuales que fortalecerán el desarrollo de habilidades y actitudes tales como: pensamiento crítico y creativo, responsabilidad ante el aprendizaje, búsqueda, organización, creación y aplicación de información, promoción del aprendizaje colaborativo y autorreflexión sobre el propio aprendizaje.

Palabras clave: trabajo colaborativo, estrategias didácticas, convivencia escolar.

\section{ABSTRACT}

The objective of the proposal is to improve collaborative work in students of General Basic Education in the Educational Unit of "Quisapincha", where you can see a lack of interest when working collaboratively, the project aims to use teaching strategies, which are essential tools for driving the educational process and the construction of new knowledge to generate a bond of good coexistence, human and

\footnotetext{
${ }^{1}$ Magister en Docencia y Currículo para la Educación Superior. Docente de la Facultad de Educación y Desarrollo Social de la Universidad Tecnológica Indoamérica. Ecuador. E-mail: eulaliabecerra@uti.edu.ec

${ }^{2}$ Magister en Gerencia y Liderazgo Educacional. Docente de la Facultad de Educación y Desarrollo Social de la Universidad Tecnológica Indoamérica. Ecuador. E-mail: gloriaendara@uti.edu.ec

${ }^{3}$ Licenciada en Educación Básica. Estudiante Universidad Tecnológica Indoamérica. Ecuador. E-mail: pomapiedad@gmail.com
} 
social relationships to achieve teamwork and harmonic groups, the research uses a qualitative-quantitative methodology, since the analysis and interpretation of the techniques and instruments such as the interview and survey, it was determined that the third year students of basic general education work individually and in isolation with absence of coexistence and values of collaboration; Therefore, it is suggested to use a manual of collaborative teaching strategies that will strengthen the development of skills and attitudes such as: critical and creative thinking, responsibility for learning, searching, organizing, creating and applying information, promoting collaborative learning and self-reflection on learning itself.

Keywords: collaborative work, teaching strategies, teamwork.

\section{INTRODUCCIÓN}

La línea de investigación de este estudio es la Innovación Educativa, para lo cual se desarrollaron y se aplicaron técnicas y actividades que se encargaron en primer lugar de acostumbrar a los niños a trabajar en equipo, luego se logró que asimilen de mejor manera los conocimientos. La mente de los niños en estas edades tiene una alta capacidad de recepción, por lo tanto, es necesario llenarla de enseñanzas y conocimientos, pero también de valores y comportamientos "humanos" que le ayudarán a desenvolverse de mejor manera en la sociedad. Se trabajó con estudiantes de tercer año de Educación General Básica de la Unidad Educativa Quisapincha, que presentaban problemas de convivencia.

El estudio se sustentó en la sublínea de investigación aprendizaje, ya que promueve la implementación de diferentes estrategias didácticas originando una interrelación de aprendizajes significativos para el niño y niña a través del trabajo colaborativo. Es una manera de fomentar en el alumno la capacidad de trabajar en equipo y de forma activa para alcanzar un mejor desempeño académico en las materias básicas.

Luego del análisis de los resultados obtenidos se detectó que el principal problema presente en las aulas es la falta de integración por parte de los estudiantes de educación básica de la Unidad Educativa Quisapincha, al momento de realizar las actividades grupales. Por esta razón, a través de las estrategias didácticas como el juego, el canto y trabajos manuales, el docente puede fomentar el trabajo colaborativo en los alumnos. Las estrategias didácticas como el juego, el canto y trabajos manuales, ayudan a que el docente pueda fomentar el trabajo colaborativo en los alumnos. Estas estrategias se aplican para que todos participen en comunidad con la finalidad de lograr una interacción grupal y alcanzar niveles de aprendizajes aceptables.

De la misma forma, el trabajo colaborativo basado en disciplina y reglas establecidas es un factor relevante en el sentido de que fomenta la participación democrática y la igualdad de condición entre un grupo de estudiantes al momento de opinar y defender puntos de vista. El respeto por la opinión ajena, también se desarrolla creando en los niños una visión de tolerancia y de aceptación de iguales. Al respecto y dentro de las políticas públicas y educativas se destaca la estipulada en el Código de la Niñez y Adolescencia (2009) en el Art. 38.- que establece los siguientes literales:

a) Desarrollar la personalidad por medio de la integración, la convivencia, los valores, la capacidad intelectual y social del niño, niña y adolescente hasta su máximo potencial, en un entorno colaborativo y afectivo: 
b) Promover y practicar el trabajo colaborativo, el respeto a los derechos humanos y libertades fundamentales, la no discriminación, la tolerancia, la valoración de las diversidades, la participación, el diálogo, la autonomía y la cooperación (p. 7).

El trabajo colaborativo y la integración social entre los niños/as, está enmarcado en la colaboración, sin discriminación alguna, respetando los derechos humanos y la práctica de valores. Son prácticas que los estudiantes deben llevar a cabo para formarse académicamente dentro de la comunidad educativa.

Así mismo, en el Plan Nacional de Desarrollo (2017) en el capítulo tercero sobre los derechos de las personas y grupos de atención prioritaria, sección quinta que habla sobre las niñas, niños y adolescentes en el Art. 44 dice que:

"Del mismo modo, se revela la necesidad de propiciar y fortalecer una educación con pertinencia cultural, lingüística y ambiental, que atienda a las necesidades educativas específicas de los pueblos y nacionalidades" (p. 56)

Los niños aprenden mucho mediante el desarrollo de sus habilidades, actitudes, hábitos y convivencia con los demás. La interacción entre ellos desarrollará el pensamiento crítico, el razonamiento y la empatía.

En la Unidad Educativa "Quisapincha" el principal problema en el aula de clases es la ausencia de colaboración para realizar una tarea o trabajo en equipo, el individualismo y egoísmo se presentan en todo momento, perjudicando de forma directa a los estudiantes en la asimilación de nuevos conocimientos.

El trabajo fue realizado con la finalidad de proponer estrategias de enseñanza específicas a los docentes como las estrategias colaborativas. Como resultado relevante de la investigación se comprobó que la aplicación de las estrategias mejora la calidad de aprendizaje y el desarrollo de las habilidades sociales y cognitivas de los niños y niñas, promoviendo la integración social en todos los miembros del equipo de trabajo.

De acuerdo con Rodríguez (2012) describe que el trabajo colaborativo es la estrategia clave en la educación de hoy. La investigación se llevó a cabo en un Centro Educativo Particular a 36 niños y niñas de 8 a 10 años; con un nivel socioeconómico medio bajo y con un alto índice de vulnerabilidad ya que los estudiantes no cuentan con los materiales y medios necesarios para un buen desempeño académico para aprender pues se observó la poca participación de maestros en el acompañamiento y compromiso para la enseñanza-aprendizaje.

Moreno (2013) desarrolló una investigación titulada "El Trabajo Colaborativo como Estrategia Didáctica para mejorar el Proceso de Enseñanza-Aprendizaje”, el estudio se realizó en una institución educativa de Buenos Aires a cien estudiantes de 12 años de edad, aquí se realizaron secciones de talleres grupales en el aula de clases durante 4 semanas, con la aplicación del estudio se obtuvo como principal resultado que el trabajo colaborativo aborda una estrategia didáctica para enriquecer el proceso de enseñanza-aprendizaje. El principal objetivo del proyecto fue implementar estrategias didácticas con el uso de herramientas didácticas a través del internet y análisis de software, el uso de estas herramientas permitió la interacción de los estudiantes desde cualquier lugar, compartiendo experiencias para fortalecer la integración de los niños y niñas en todas las edades escolares.

Podestá (2016) da conocer en su investigación sobre el trabajo colaborativo entre docentes realizada en el Congreso Iberoamericano de Ciencia, Tecnología, 
Innovación y Educación, donde se llevó a cabo una formación académica a los docentes en temas relacionados a estrategias pedagógicas y su relación con la utilización de las TIC, con el objetivo de promover nuevos saberes, habilidades y actitudes a través de las distintas competencias de cada miembro del grupo. Como resultado de este trabajo se mejoró los modelos de enseñanza, la interacción y el aprendizaje colectivo, que es primordial para la asimilación de nuevos conocimientos.

Según Moreno \& Velázquez (2017) en su estudio sobre la Estrategia Didáctica para Desarrollar el Trabajo Colaborativo, menciona que el uso de las estrategias didácticas como juegos y canciones, contribuyen a que los niños vayan despertando el interés por interactuar y trabajar en equipo además contribuye al fortalecimiento emocional y afectivo entre los docentes y los estudiantes.

En función al párrafo anterior se puede manifestar que las estrategias didácticas fortalecen a los estudiantes para obtener los conocimientos de manera más rápida y sencilla con materiales y métodos adecuados según el tema que se vaya a tratar, permitiendo a los educandos una integración social dentro y fuera de la institución, al inicio o al final de la jornada, potenciando el trabajo colaborativo para alcanzar los objetivos propuestos.

Por otro lado, Silva (2005) manifiesta que el trabajo colaborativo ayuda a mejorar la convivencia entre estudiantes, permite fortalecer la integración social y aprenden a valorar el trabajo en equipo. Por esta razón, es fundamental diseñar diferentes estrategias didácticas, mediante la formación de pequeños grupos de trabajo, donde cada uno sea responsable de la tarea encomendada para lograr la meta común.

\section{METODOLOGÍA}

La presente investigación tiene un enfoque cualitativo, por cuanto se aplicó una encuesta (a los docentes) con una serie de procesos de manera secuencial para la recolección de los datos y su respectiva interpretación. Además, se aplicó las fichas de observación (a estudiantes) las mismas que permitieron conocer directamente el comportamiento de cada uno, así como sus fortalezas y debilidades que presentan al momento de trabajar colaborativamente.

Según Tamayo (2002) menciona que la investigación descriptiva permite realizar una descripción, así como un registro, análisis e interpretación de la naturaleza actual y la composición de procesos o fenómenos. Según esta descripción la presente investigación es descriptiva porque se partió del análisis de la problemática, que es la escasa utilización de estrategias didácticas para poder fortalecer el trabajo colaborativo en los estudiantes de tercer año de Educación General Básica de la Unidad Educativa "Quisapincha", al final se obtuvieron resultados de las observaciones de las conductas y las características de cada estudiante.

Silva (2014) menciona que los estudios explicativos parten de problemáticas o dificultades bien detalladas en las cuales es ineludible el conocimiento de relaciones causa- efecto. En este tipo de estudios es indispensable explicar por qué ocurre un fenómeno y en qué condiciones se manifiesta, o por qué se relacionan dos o más variables.

Tomado la expresión de Silva, se puede decir que esta investigación es explicativa debido a que existe un problema (causa) la escasa aplicación de estrategias didácticas para la integración en la sala de clases por parte del docente 
provoca que el estudiante no trabaje colaborativamente (efecto). Los resultados permitieron establecer conclusiones y recomendaciones que ayudan a corregir las prácticas docentes y a tener una amplia comprensión del problema; con la ayuda de la información científica se pudo diseñar estrategias didácticas más factibles para los estudiantes de tercer año de Educación General Básica de la Unidad Educativa "Quisapincha".

La población con la que se trabajó fue de 30 estudiantes del tercer año de EGB (Educación General Básica), y 3 docentes de la Unidad Educativa "Quisapincha", a quienes se les aplicó una ficha de observación y una encuesta respectivamente.

\section{RESULTADOS Y DISCUSIÓN}

Luego de la aplicación de los instrumentos de investigación a los estudiantes se obtuvo que la mayoría de ellos no trabajan en equipo ni dentro ni fuera del aula, no muestran interés a la hora de trabajar en equipo, tampoco admiten sus errores al momento de desarrollar actividades y no aportan con toda su capacidad y creatividad para construir nuevos conocimientos, esto hace que los estudiantes no se sientan felices cuando trabajan en equipo, por lo que les causa dificultad a la hora de integrarse de forma colectiva, hay poco respeto y compañerismo al momento de desarrollar actividades en equipo, esto ocasiona dificultades en el proceso de enseñanza aprendizaje. Por otro lado, la mayoría de los estudiantes no aplican valores de convivencia estudiantil. Los resultados revelan que la falta de estrategias didácticas da como resultado bajo rendimiento académico y un comportamiento poco satisfactorio, como a continuación se detalla en la Figura 1:

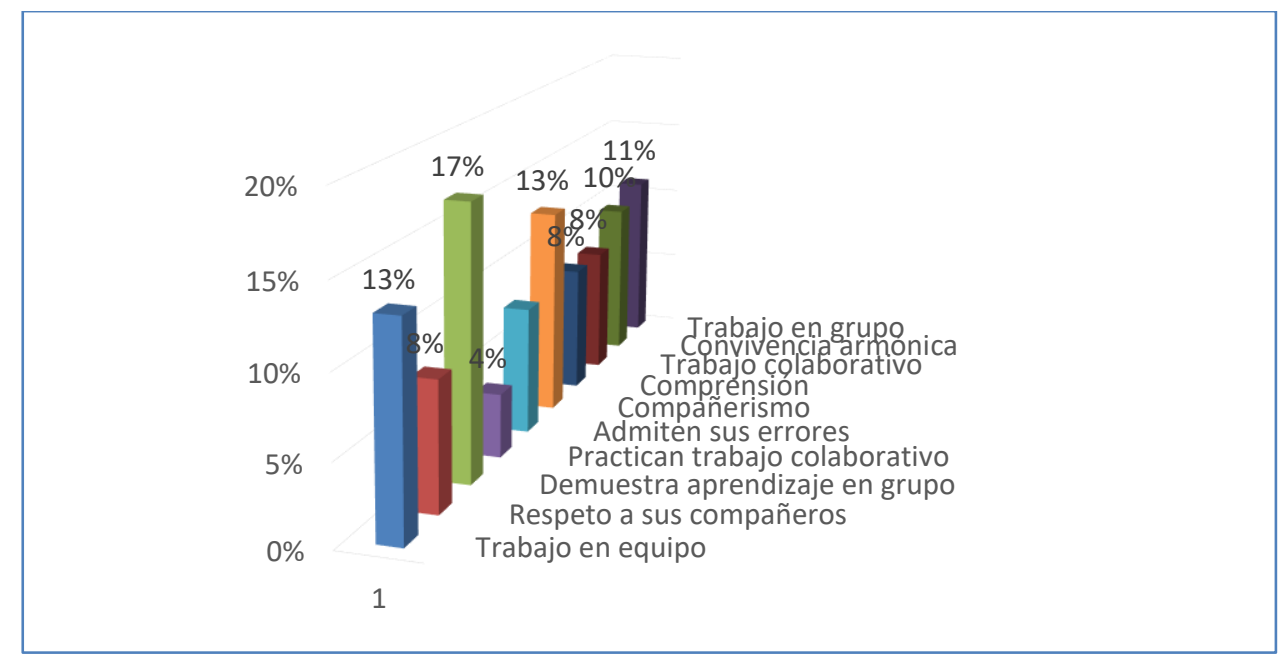

Figura 1: Comportamiento de los estudiantes en función de las estrategias didácticas

El análisis e interpretación de los resultados determina que el nivel de trabajo colaborativo de los estudiantes en el área de Lengua y Literatura es bajo, el docente desconoce estrategias y recursos para realizar las actividades que beneficien la integración de los educandos, por lo que es necesario diseñar estrategias didácticas para mejorar el trabajo colaborativo en los estudiantes del tercer año de Educación General Básica de la Unidad Educativa "Quisapincha". Estrategias didácticas que 
orienten a los maestros a mejorar el trabajo colaborativo en los alumnos, con la aplicación de actividades que incluyen juegos y canciones, en donde el estudiante y docente propicien un ambiente colaborativo y participativo.

En la Unidad Educativa "Quisapincha" el principal problema en el aula de clases es la ausencia de colaboración para realizar una tarea o trabajo en equipo, el individualismo y egoísmo se presentan en todo momento, perjudicando de forma directa a los estudiantes en el proceso de enseñanza aprendizaje.

Para la validación del trabajo se tomó en cuenta el criterio de dos especialistas expertos en el área de Lengua y Literatura, los resultados se presentan a continuación en la siguiente tabla:

Tabla 1: Validación de especialistas

\begin{tabular}{lccc}
\hline \multicolumn{1}{c}{ Criterios } & MA & PA & A \\
\hline Estructura de la propuesta & $100 \%$ & \\
\hline $\begin{array}{l}\text { Claridad de la redacción } \\
\text { (lenguaje sencillo) }\end{array}$ & $100 \%$ & \\
$\begin{array}{l}\text { Pertinencia del contenido de la } \\
\text { propuesta }\end{array}$ & $100 \%$ \\
$\begin{array}{l}\text { Coherencia entre el objetivo } \\
\text { planteado e indicadores para } \\
\text { medir resultados esperados. }\end{array}$ & $100 \%$ & \\
\hline
\end{tabular}

\begin{tabular}{ll}
\hline \multicolumn{2}{c}{ TOTAL 100\% } \\
\hline $\begin{array}{l}\text { Fuente: Manual de } \\
\text { Universidad }\end{array}$ \\
$\begin{array}{l}\text { Indoamérica, 2019 2.0, } \\
\text { Tecnológica }\end{array}$
\end{tabular}

\section{Estrategias para el trabajo colaborativo}

Las estrategias didácticas permiten mejorar el trabajo colaborativo con actividades que ayudan al desenvolvimiento, integración, colaboración, convivencia armónica entre los grupos de participantes dejando de lado el individualismo y egoísmo, adentrándose a la participación integral de todos, estas actividades como el arco iris de colores, la torre más alta, la trama del ovillo entre otras, son actividades que fomentan el interés y la motivación para el aprendizaje. Las actividades de cada estrategia se encuentran explicadas detalladamente y con ejemplos que pueden adaptarse en las horas de clase ya sea dentro o fuera del aula; dependerá de la creatividad del docente para usarlas en el fortalecimiento del trabajo colaborativo.

Cada estrategia está estructurada de la siguiente manera:

Índice: debe consignarse todos los títulos.

Presentación: se precisa el uso y función del manual de estrategias didácticas.

Objetivos generales: permiten al participante identificar los requerimientos conceptuales, procedimentales y actitudinales básicos.

Esquema-resumen de contenidos: presentar en forma resumida las estrategias.

Desarrollo de contenidos: aquí se hace una presentación general de la temática, ubicándola en su campo de estudio. 
Premisas para su implementación

Para la implementación de las estrategias se desarrollarán las siguientes actividades:

- Sensibilización a la comunidad educativa sobre la importancia de la utilización de estrategias didácticas de trabajo colaborativo como una herramienta didáctica dentro del aula para desarrollar la convivencia integral del estudiante.

- Planeación de aplicación en la institución educativa.

- Identificación de grupos de trabajo para la organización.

- Monitoreo y evaluación del producto.

- Reporte de impacto del manual de estrategias en la disminución o eliminación del problema.

A continuación, se presenta ejemplos de las estrategias.

Arcoíris de colores

Objetivo: Favorecer la colaboración y la ayuda mutua entre compañeros para el desarrollo del aprendizaje.

Participantes: Niños y niñas

\section{Materiales:}

2 paracaídas

1 pelota pequeña

Área: Matemáticas

Tiempo Estimado: 30 minutos

Motivación: canción "Rin rin"

En el coche de mamá

nos iremos a pasear.

Vamos de paseo

$\mathrm{Pi}$, pi rin rin.

En un coche viejo

$\mathrm{Pi}$, pi rin rin.

Pero no me importa

$\mathrm{Pi}$, pi rin rin.

Porque llevo tanta torta

$\mathrm{Pi}$, pi rin rin.

\section{Procedimiento:}

El docente sale al patio y presenta un paracaídas construido de cartón

Al abrir el paracaídas se colocan los estudiantes al rededor

El maestro/a da indicaciones generales sobre el juego, si el paracaídas cae cerca de un estudiante este participará

Comienza lanzando el paracaídas desde el centro, el estudiante que le corresponde manifiesta el tema interesante. 
El estudiante del centro lanza el paracaídas a otro compañero que desea responder a la pregunta propuesta por su compañero.

Todos participan con respuestas diferentes aportando cada uno con su conocimiento individual que a la larga llegara a ser un juego colaborativo ya que todos trabajan aportando ideas que fortalecerá $n$ el conocimiento de todos.

\section{Evaluación:}

Los criterios de evaluación se toman en cuenta en base a las respuestas que emita el estudiante a cada pregunta, esto varía según tema a tratar en la clase

\section{La torre más alta}

Objetivo: Lograr el trabajo colaborativo mediante una comunicación efectiva entre los estudiantes. A través de juegos.

Participantes: Niños y niñas

Materiales:

1 funda de tallarín

1 cinta de papel

1 mesa

Área: Matemáticas

Tiempo Estimado: 30 minutos

Motivación: Canción "La torre más alta"

Vivo en una torre, muy alta, muy alta,

Con ventanas y tejados hermosos,

Paredes lucidas y puertas altas,

Y una vista espectacular,

Una recamara encantadora donde duermo yo.

\section{Procedimiento:}

El profesor/a divide el grupo en tres o cuatro subgrupos., luego el maestro en el aula presenta las instrucciones para poder realizar el juego colaborativo y los materiales que serán utilizados. Luego distribuye el material en cada grupo (los tallarines, cinta de papel,) cuidando que sea en proporción igualitaria para cada grupo. Todos los estudiantes deberán armar la torre más alta con un cierto número de tallarines en un tiempo determinado de esa manera trabajan de forma colaborativa para llegar a la actividad planificada, logrando mantener un ambiente acogedor e integrando a todos los participantes dentro del grupo del trabajo.

\section{Evaluación:}

Una vez concluido el tiempo determinado se selecciona un coordinador para que pueda exponer cuales fueron sus fortalezas y debilidades al trabajar de forma colaborativa, de acuerdo con los parámetros de desarrollo de cada estrategia.

\section{La trama del ovillo}

Objetivo: Favorecer la integración grupal para que exista una buena convivencia entre todos los miembros del equipo.

Participantes: Niños y niñas

Materiales:

Lana de color

Área: Matemáticas 
Tiempo Estimado: 30 minutos

Motivación: Canción "La araña lanuda"

La araña lanuda, salió a caminar

Caía la lluvia y tuvo que nadar

El sol salió, y la arena se seco

Y la araña peluda de nuevo se escondió.

\section{Procedimiento:}

Los estudiantes se ubican en un lugar adecuado de forma circular, se entrega a un participante la lana este debe decir un numero secuencial, el otro estudiante toma la punta de la lana y lo entrega a otro compañero, quien a su vez va a seguir la secuencia del número que dicto el primer participante.

La acción será repetida hasta que el estudiante forme un enlace con la lana, en forma de una tela araña, de la misma manera debe regresar al que le envió nombrando el número que dijo anteriormente el compañero en forma reversa hasta que la lana llegue a formarse un ovillo y regrese la punta al estudiante que inicio el juego.

\section{Evaluación:}

Se toma en cuenta la colaboración y entusiasmo de cada participante al momento de integrarse en la actividad presentada.

\section{Encajando la figura}

Objetivo: Desarrollar la imaginación y la creatividad dentro de un grupo de trabajo Técnicas e instrumentos.

Materiales:

Hojas impresas

Tijera

Goma

cartulina

Área: Matemáticas

Tiempo: 30 minutos

Motivación: Canción" Periquito"

Periquito el bandolero

Se metió en un sombrero

El sombrero era de paja

Se metió en una caja

La caja era de cartón

Se metió en un cajón

El cajón era de pino

se metió en un pepino

El pepino maduro y Periquito se salvó.

\section{Procedimiento:}

El docente presenta las piezas de distintas figuras, luego se forma grupos de 7 participantes, se entrega el material necesario a cada grupo. Cuando el docente de una señal los alumnos tendrán que formar los grupos, se escogerá un líder para que 
guie la actividad a elaborar, luego recortaran las hojas de trabajo por consiguiente pintara las piezas y arman la figura que se les indica. Finalmente se expone el trabajo dando a conocer las experiencias que ellos tuvieron al trabajar en grupos.

\section{Evaluació:}

Equilibrar una determinada figura, para luego comparar las piezas y armarla en grupo de acuerdo con el modelo indicado y así tener una relación armónica entre los estudiantes.

\section{CONCLUSIONES}

Las investigaciones pertinentes sobre estrategias didácticas permiten mejorar el trabajo colaborativo de los estudiantes del tercer año de educación general básica de la Unidad Educativa "Quisapincha", esto se evidencia al aplicar los juegos colaborativos didácticos que sirvieron para fortalecer la integración social y mejorar las relaciones entre los estudiantes.

Las estrategias didácticas de trabajo colaborativo juntamente con la evaluación de actividades ayudan a los estudiantes a la cooperación activa, para fomentar la convivencia en los grupos de trabajo con la finalidad de alcanzar un aprendizaje significativo.

\section{REFERENCIAS BIBLIOGRÁFICAS}

Congreso Nacional (2009). Codigo de la Niñez y Adolescencia. Quito: Repúbica del Ecuador (p. 7)

Consejo Nacional de Planificación (2017). Plan Nacional de Desarrollo. Toda una Vida. Quito, Ecuador. (p. 56). Recuperado de http://www.planificacion.gob.ec /wpcontent/uploads/downloads/2017/10/pnbv-26-oct-

final_0k.compressed1.pdf

Moreno, E. (2013). El Trabajo Colaborativo como Estrategia para Mejorar el Proceso de Enseñanza-Aprendizaje. Buenos Aíres.

Podestá. (2014). Buenos Aíres: ISBN: 978-84-7666-210-6.

Podestá, P. (2016). El trabajo colaborativo entre docentes: experiencias en la Especialización Docente Superior en Educación y TIC. Buenos Aíres: Instituto Nacional de Formación Docente.

Rodríguez, I. (2012). Trabajo colaborativo: Estrategia clave en la educación de hoy. Buenos Aires.

Silva, C. (2016). La formulación de problemas de investigación en la educación. Rev Cub Cardiol Cir Cardiovasc.

Tamayo, M. (2002). El proceso de la Investigación Científica. Revista Noriega Editores. Año. México. Edición cuarta

Vázquez, J. M. (2015). Trabajo colaborativo: acciones para su implementación en la gestión del talento humano y la docencia. Multiversidad Management, (19) pp. 36-42. 